\title{
Systematik der Restrukturierung des Fertigungskomplexes eines mittelståndischen Gerätebetriebes
}

\author{
Dipl.-Ing. (FH) Astrid Guhl
}

\section{Kurzcharakteristik}

Hauptinhalt des vorliegenden Beitrages ist es, die gewonnenen Erkenntnisse bei der Anwendung eines systematischen Planungsablaufes in der Praxis darzustellen und dadurch ein Beispiel für die Bearbeitung von fabrikplanerischen Aufgaben zu geben. Im Mittelpunkt der hier vorgestellten Planungsaufgabe stehen die Schaffung eines guinstigen Materialflusses und die Bedarfsplanung hinsichtlich erforderlicher Betriebsmittel und Flächen. Zur Lösung der Problemstellung werden die einzelnen Phasen des systematischen Planungsablaufes durchlaufen. Dadurch wird die funktionale Verkniipfung und die logisch-zeitliche Folge der wesentlichen Planungsschritte verdeutlicht. Somit ist eine wichtige Voraussetzung fuir die auf die Gesamtzielsetzung ausgerichtete Koordination der erforderlichen Teilaufgaben gegeben.

Ausgehend von der Ist-Zustands-Analyse eines bestehenden Fertigungskomplexes werden der Inhalt der Planungsaufgabe erläutert und die grundsätzliche Vorgehensweise bei der systematischen Fabrikplanung detailliert dargestellt. Durch ein Beispiel aus der Praxis soll die Umsetzung dieses Planungsablaufes demonstriert und die sich daraus ergebenden Veränderungen erläutert werden. Anschließend wird der neu strukturierte Fertigungskomplex bewertet.

\section{Analyse des Ist-Zustandes}

Bei der hier vorgestellten Aufgabe handelt es sich um eine Um- bzw. Erweiterungsplanung. Somit kann auf die Ermittlung und Analyse eines bereits bestehenden Zustandes als Ausgangsbasis für die Planung zuruickgegriffen werden. Der Untersuchungsumfang wurde auf das für die Aufgabenstellung notwendige und wirtschaftlich vertretbare Maß beschränkt.

Zur Bewertung des Ist-Zustandes des Fertigungskomplexes waren in Abhängigkeit der Zielsetzung des Projektes folgende Untersuchungsbereiche relevant:

- allgemeine Informationen (Produktionsprogramm, Aufbauorganisation, Personal- und Arbeitsplatzstruktur),

- Betriebsanlagen und -einrichtungen (Gebäude, Fertigungs- und Transportmittel, Lagereinrichtungen),

- Erzeugnisgestaltung (Erzeugnisspektrum, Entwicklung, Konstruktion),

- Fertigung (Art, Prinzip und Verfahren der Fertigung, Arbeitsvorbereitung),

- Fertigungsfluß (Material-, Informations-, Energie- und Personenfluß).

Zur Aufnahme des Ist-Zustandes wurde sowohl die direk- te als auch die indirekte Methode der Datenerfassung angewandt. Oftmals erwiesen sich die vorhandenen betrieblichen Unterlagen als unzureichend und mußten entsprechend uiberarbeitet werden.

Das verwendete Produktionsprogramm umfaßte einen Planzeitraum von 3 Jahren und stellte somit eine mittelfristige Programmplanung dar.

- Stiickzahl

- Durchschnittspreis

- Umsatz

- Materialeintrag

Diese Werte bildeten die Basis für die Kapazitäts- und Bedarfsplanung sowie für die Materialfluß- und Layoutoptimierung. Zur Auswertung der Planungsdaten wurde die PQ-Analyse (Produkt-Quantum-Analyse) verwendet. Durch graphische Gegenuiberstellung der oben genannten Beurteilungskriterien ermöglicht die PQ-Analyse eine kritische Analyse des Produktionsprogramms und bildet gleichzeitig eine wichtige planerische und unternehmerische Entscheidungshilfe. Es muß jedoch darauf hingewiesen werden, daß das Produktionsprogramm einer Vielzahl von produkt-, markt- und betriebswirtschaftlichen Einfluissen unterliegt und die Planungsdaten nur als An-nahmewerte zu verstehen sind.

Nach der Analyse der weiteren Untersuchungsbereiche schloß sich eine Bewertung des Ist-Zustandes an. Um dabei eine objektive Aussage zu bekommen, ist es ratsam, zunächst Beurteilungskriterien festzulegen. Diese können sich nach allgemeinen Leitsätzen zur Materialflußgestaltung und in der Produktion üblichen modernen Gestaltungstechniken richten.

Die Beurteilung erfolgte nach technischen und organisatorischen Gesichtspunkten, wobei die vorhandenen Schwachstellen, aber auch Vorteile des Ist-Zustandes betrachtet wurden. Im Folgenden sind die wichtigsten Ergebnisse der Ist-Zustands-Analyse genannt:

- hohe Materialflußvernetzung,

- keine optimale Raumnutzung in der Produktion und im Lager,

- Hauptlager und Fertigung nicht in einer Ebene,

- dezentrale Lagerung von Baugruppen,

- keine räumliche Trennung von Lager und Verpackung,

- Lagerwesen der Materialflußintensität angepaßt,

- kein einheitliches Förderhilfsmittelsystem.

\section{Notwendigkeit zur Restrukturierung - Planungsaufgabe}

Die Neugestaltung des hier als Praxisbeispiel dienenden Fertigungskomplexes eines Gerätebriebes hatte ver- 
schiedenen Ursachen, die in [1] detailliert aufgeführt sind. Zum einen erforderte die Entwicklung neuer Produkte und die damit verbundenen Technologieveränderungen eine Umstrukturierung der bisherigen Fertigung. Zum anderen erwies sich die existierende Produktionsstrategie als ungeeignet (siehe Abb. 2). Danach erschwert sich durch die vorhandende Mehrfachlagerung die Lagerbestandsiiberwachung, und Transportgeräte werden nicht optimal ausgelastet.

Fertigung und Lager befinden sich in unterschiedlichen Gebäudeebenen, wodurch sich die Transportwege und der Transportaufwand erheblich erhöhen. Die räumlich von der Verpackung nicht getrennte Lagerung der Fertigerzeugnisse erhöht die ungewollte Einwirkung von Staub- und Schmutzpartikeln.

Ein weiterer wichtiger Entscheidungsfaktor zur Restrukturierung war das Vorhaben der Firma, die gesamte Produktion in ein neues Gebäude umzulagern.

Im Mittelpunkt der Planungsaufgabe stand die Schaffung eines guinstigen Produktions- und Fertigungsflusses, wobei auf die Materialflußgestaltung besonderer Wert zu legen war. Die Optimierunng des Materialflusses beinhaltete folgende Zielsetzung:

- Verminderung der Durchlaufzeit der Produkte, d. h. Verkuirzung der möglichen Lieferzeiten und somit Einhaltung der Termintreue,

- Senkung der Bestände, d. h. Reduzierung der Kapitalbindungskosten (Lager- und Verwaltungskosten),

- Verbesserung der Flächen- und Raumnutzung, d. h. Senkung der Kosten für Flächennutzung (Grundstiickskosten),

- Steigerung der Auslastung von Transport- und Lagereinrichtungen.

Desweiteren war bei der Planung auf gute Erweiterungsmöglichkeiten zu achten. Die vorhandenen Ressourcen an Produktionsmitteln wurden in die Planung mit einbegriffen. Begleitende Kostenbetrachtungen bleiben späteren Untersuchungen vorbehalten.

\section{Systematik des Planungsablaufes}

Für die Lösung komplexer Fabrikplanungsaufgaben ist die Erarbeitung und konsequente Einhaltung eines systematischen Planungsablaufes unter Beachtung der allgemeinen Planungsgrundsätze unerläßlich. Das hier dargestellte Grundprinzip (siehe Abb. 1) stiitzt sich im wesentlichen auf die in |2| erläuterten Stufen eines systematischen Planungsablaufes.

Im Folgenden sollen die Inhalte der einzelnen Planungsphasen näher betrachtet werden.

\section{Aufgabenstellung/Zielsetzung}

Die Aufgabenstellung ist so exakt und eindeutig wie möglich zu formulieren, um eventuell auftretende Unklarheiten von vornherein zu vermeiden. Dabei sollte die Zielsetzung zwar anspruchsvoll, aber auch in einem realisierbaren Rahmen festgelegt werden.

Wichtig ist auch die Bestimmung der Mitarbeiter, die an dem Planungsprojekt mitwirken. Dabei kann sich diese Projektgruppe aus Mitarbeitern der verschiedenen Betriebsbereiche zusammensetzen. Oftmals arbeiten auch betriebsfremde Planer an einem Projekt mit, deren Erfahrungen im Umgang mit modernen Planungsmethoden genutzt werden können.

Aufnahme und Analyse des Ist-Zustandes

Primäres Ziel der Ist-Zustands-Ermittlung ist die Schaffung von Ausgangsdaten für die Planung. Bereits bei Aufnahme des Ist-Zustandes können technische, wirtschaftliche und organisatorische Schwachstellen aufgedeckt werden, die wiederum Grundlage bei der Neugestaltung sind. In den meisten Fällen kann bei der Ermittlung und Analyse auf einen bestehenden Zustand zurïckgegriffen werden (Um- oder Erweiterunsplanung). In jedem Fall sollte der Untersuchungsrahmen auf das für die Aufgabenstellung benötigte Maß festgelegt sein.

Die Aufnahme des Ist-Zustandes wird nach unterschiedlichen Methoden durchgefuihrt. Die direkte Datenerfassung erfolgt bei laufender Produktion durch Befragung der Mitarbeiter, durch Beobachtung oder Planwertverfahren. Dem gegenuiber stuitzt sich die indirekte Methode der Datenerfassung auf vorhandene betriebliche Unterlagen, wie z. B. Lage- und Bebauungspläne, Produktions- und Absatzstatistiken, Maschinenkarteien und Fertigungsunterlagen. Durch den Ruickgriff auf betriebliche Unterlagen kann der Zeitaufwand im Rahmen der Ist-Zustands-Analyse erheblich reduziert werden. Voraussetzung ist aber die Überprüung der betrieblichen Daten auf Aktualität und Vollständigkeit. In der Praxis sind die im Betrieb vorhandenen Unterlagen sehr oft unzureichend und für spezielle Aufgabenstellungen nicht geeignet. Sie muissen dann ggf. vervollständigt oder erweitert werden.

Die Aufnahme und Bewertung des Ist-Zustandes stellt den wichtigsten und zugleich arbeitsintensivsten Planungsschritt der Vorarbeiten dar. Das Ergebnis jeder Pla-

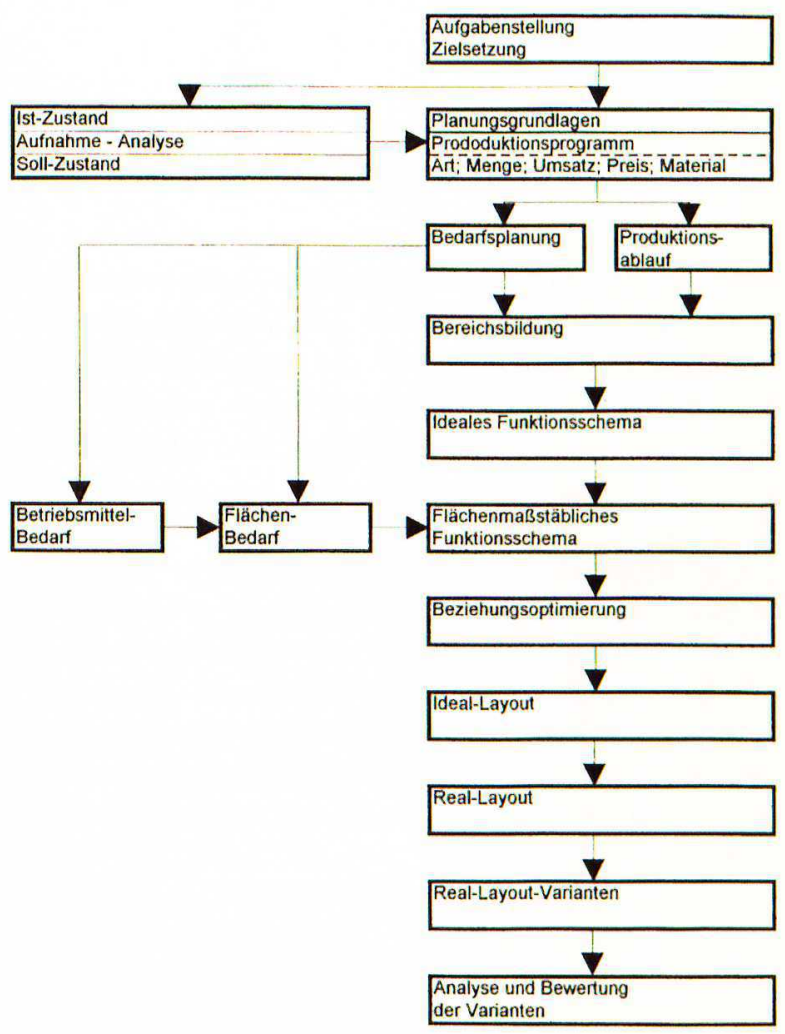

Abb. 1 Systematischer Planungsablauf nach [2]. Grundprinzip 
nung hängt aber im entscheidenden Maße von der Qualität der Ausgangsdaten ab. Daher ist dieser Planungsstufe besondere Aufmerksamkeit zu schenken.

Nach der Aufnahme des Ist-Zustandes schließt sich die Bewertung der gewonnenen Daten an, nach der eine Schwachstellenanalyse erstellt wird. Diese Analyse bildet die Basis des Soll-Zustandes.

\section{Planungsgrundlage}

Die wichtigste Planungsgrundlage stellt das Produktionsprogramm dar. Dort wird festgelegt, welche Produktkarten und -mengen innerhalb einer Zeitperiode in einem Unternehmen gefertigt werden. Damit sind im wesentlichen auch die Produktionstechnologien und -verfahren bekannt. Diese Daten sind Grundlage zur Bedarfsermittlung.

\section{Bedarfsplanung}

Ausgehend von dem zu Grunde liegenden Produktionsprogramm erfolgt im Rahmen der Bedarfsermittlung eine Abschätzung des voraussichtlichen Bedarfes an Betriebsmitteln, Personal, Fläche und Energie. Diese vier Bedarfsarten stellen das zu verplanende Potential dar, das zu einer dem vorgegebenen Produktionsprogramm entsprechenden Fertigung fuihrt. Sind bereits Betriebsmittel vorhanden und genuigen diese den geforderten Technologien, sind sie, unter Beachtung der Wirtschaftlichkeit, in das neue Produktionskonzept mit einzuplanen. Sowohl bei der Betriebsmittelbedarfsplanung als auch bei der Flächenbedarfsplanung ist der Aspekt der „Erweiterungsplanung“" zu beachten.

\section{Bereichsausbildung}

Die Bereichsbildung ist der Beginn der Layoutplanung. Durch Zusammenfassung von funktionell gleichartigen oder eng miteinander verknüpften Arbeitsvorgängen werden sogenannte Funktionseinheiten (Bereiche) gebildet. Wesentliche Kriterien sind dabei die Gleichartigkeit technologischer oder raumqualitativer Anforderungen sowie organisatorische Zusammenhänge. Diese Kriterien werden durch die Festlegung von Festlegungsart und -prinzip bestimmt.

In Abhängigkeit der Planungsaufgabe kann die Größe der Funktionseinheiten ermittelt werden (Arbeitsplatz bzw. Maschinengruppe, Abteilung, Werkstatt). Es empfiehlt sich aber die Bildung kleinerer Funktionseinheiten, da sich dadurch individuellere Gestaltungmöglichkeiten bieten.

\section{Ideales Funktionsschema}

In einem idealen Funktionsschema wird die ablauf- und funktionsgerechte Zuordnung der Funktionseinheiten bzw. Bereiche dargestellt. Dabei soll diese Darstellungsform bewußt allein die ideale Zuordnung verdeutlichen und keine Ruicksicht auf reale Gegebenheiten und Randbedingungen (z. B. Flächengröße, Gebäudeabmessung) nehmen. Auch die Intensität der Materialflußbeziehungen zwischen den einzelnen Bereichen findet hier noch keine Beachtung. Der Hauptzweck des idealen Funktionsschemas liegt darin, eine uibersichtliche Vorstellung des Produktionsablaufes mit den darin integrierten Funktionen zu erhalten.

\section{Flächenmaßstäbliches Funktionsschema}

Bezugnehmend auf den unter dem Punkt Bedarfsplanung ermittelten Flächenbedarf werden die gebildeten Funktionseinheiten maßstäblich aufgezeichnet. Die endgültige Flächenform ist im flächenmaßstäblichen Funktionsschema in keiner Weise vorbestimmt. Die Anordnung der einzelnen Flächeninhalte zueinander sollte dabei dem idealen Funktionsschema weitestgehend entsprechen. Durch das flächenmaßstäbliche Funktionsschema sind die bestehenden Größenverhältnisse und die funktionsgerechte Zuordnung der einzelnen Bereiche anschaulich dargestellt.

\section{Beziehungsoptimierung}

Um eine weitere Optimierung bei der Anordnung der Funktionseinheiten zu erreichen, werden diese nach ihrer funktionellen Abhängigkeit in einer Beziehungsmatrix dargestellt.

Da in der Regel die Materialflußbeziehungen Priorität besitzen, geschieht dies iiberwiegend in Form von einer Von-Nach-Matrix (Transportmatrix).

\section{Ideal-Layout}

Mit dem idealen und flächenmaßstäblichen Funktionsschema sowie der materialflußseitigen Optimierung der Funktionseinheiten kann erstmalig im Ablauf der Planung ein vollständiges und flächenmaßstäbliches Layout entwickelt werden. Durch eine kompakte Zusammenführung der Funktionseinheiten unter möglichst weitgehender Einhaltung der idealen Zuordnungen erhält man das Ideal-Layout. Dieses wird ohne Rücksicht auf technische und wirtschaftliche Beschränkungen und unbeeinflußt von vorhandenen Restriktionen entwickelt. Dieser Idealplan stellt einen objektiven Maßstab für die nachfolgende reale Lösung dar und läßt deren Unzulänglichkeiten erkennen.

\section{Real-Layout}

Der bisher erstellte Ideal-Zustand der Anordnung der einzelnen Fertigungsbereiche zueinander muß im Rahmen der Realplanung an vorhandene bauliche Gegebenheiten und Restriktionen angepaßt werden. Restriktionen ergeben sich dabei meist durch zur Verfügung stehende Grundstiicke (Neu- bzw. Erweiterungsplanung) bzw. durch die verplanbare Hallenfläche und -höhe (Umbzw. Erweiterungsplanung). Oft liegen mehrere Real-Layout-Varianten vor, die mit Hilfe eines geeigneten Verfahrens (z. B. Punktbewer-tungsschema) entsprechend bewertet werden. Zum systematischen Planungsablauf gehört natürlich eine begleitende Kosten- und Terminplanung, die in jeder Planungsphase durchgefuihrt bzw. uiberprift werden sollte. Eine Planung ist umso effektiver, je günstiger das Verhältnis zwischen Planungsaufwand und Planungsergebnis ist.

\section{Praxisbeispiel für die Umsetzung des systematischen Planungsablaufes}

Die Umgestaltung des Fertigungskomplexes eine Gerätebetriebes soll als Beispiel fuir die Anwendung des im vorangegangenen Kapitel erläuterten systematischen 
Planungsablaufes in der Praxis dienen. Die Zielstellung liegt dabei in der Erstellung eines Ideal-Layouts und der Erarbeitung allgemeiner Gestaltungskriterien, die bei einer Restrukturierung zu beachten sind.

\subsection{Vorarbeiten}

Ausgehend von der unter Punkt 2 erläuterten Planungsaufgabe und des zu Grunde liegenden Produktionsprogramms wurde eine Produktanalyse durchgefuihrt.

Der Gerätebetrieb hat eine sehr umfangreiche Produktpalette. Es werden 8 verschiedene Grundtypen gefertigt, von denen es in Abhängigkeit bestimmter Parameter jeweils noch Sonderausfiihrungen gibt.

Die Fertigung jedes Erzeugnisses läßt sich prinzipiell in 2 Abschnitte gliedern:

- kundenanonyme Aufträge im Bereich der Serienfertigung,

- kundenspezifische Aufträge im Bereich der Einzelfertigung.

Als Fertigungsprinzip liegt die typische Werkstattfertigung (Verrichtungsprinzip) vor: Grundlage der weiteren Planung bildeten die Arbeitsablaufpläne der Grundtypen. Da sich die im Unternehmen vorhandenen Unterlagen als unzureichend erwiesen, war eine Überarbeitung der Arbeitsablaufpläne notwendig.

Im nächsten Schritt wurde eine Übersicht erstellt, in der die einzelnen Arbeitsvorgänge den jeweiligen Erzeugnissen zugeordnet sind. Somit war zu erkennen, welche Arbeitsgänge von welchen Produkten durchlaufen werden. Desweiteren wurden den Arbeitsgängen die entsprechend benötigten Betriebsmittel, die Grundfläche des Beriebsmittels, die erforderliche Medienversorgung und die Ruist- und Bearbeitungszeiten für das jeweilige Erzeugnis zugeordnet. Auf Grund der deutlichen Unterschiede im technologischen Bearbeitungsablauf der Produkte ergibt sich keine generelle Reihenfolge der Bearbeitungsstufen. Deshalb wurden Betriebsmittel zu organisatorisch gegeneinander abgegrenzten Einheiten zusammengefaßt. Bestimmend wirkten dabei geforderte raumqualitative Bedingungen.

Eine weitere wichtige Planungsvoraussetzung war der Entwurf der Produtkionslogistik. Hier wurde zunächst eine logistikgerechte Produktionsstrategie festgelegt. Ausgehend von dem in Punkt 2 vorgestellten Ist-Zustand der Produktionsstrategie wurden verschiedene Modelle erarbeitet und bewertet. Danach erweist sich das Prinzip der zentralen Lagerung (Abb. 2) unter folgenden Bedingungen als geeignet:

- Auslagerung der Verpackung aus dem Zetnrallager als räumlich getrenntes Warenausgangslager, um vermehrte Staub- und Schmutzeinwirkung auf die zu lagernden Enderzeugnisse zu vermeiden,

- abgeschirmte Lagerung von Zwischenfabrikaten aus der Produktion zur Verhinderung des Einwirkens von Umwelteinflüssen,

- direkte Anbindung des Zentrallagers an die Produktion zur Senkung des erforderlichen Transportaufwandes.

Desweiteren wurden Kenngrößen für eine logistikgerechte Ablauforganisation und Materialflußgestaltung festgelegt.

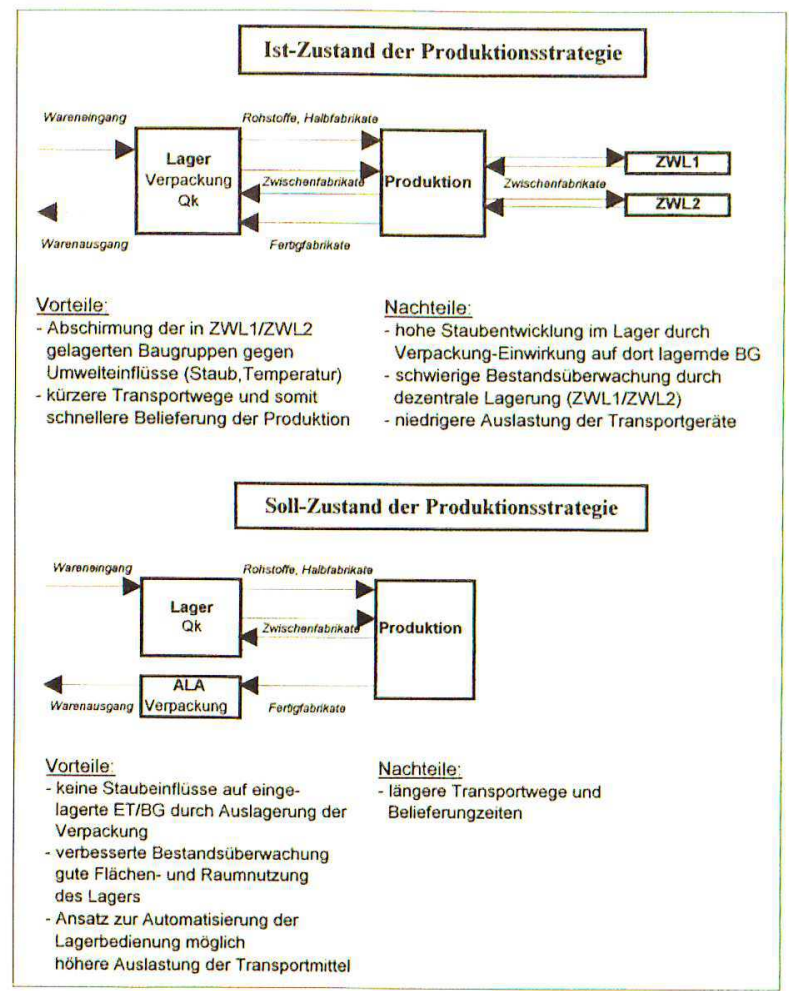

Abb. 2 Logistikgerechte Produktionsmittelstrategie

\subsection{Bedarfsplanung}

Die Grundlage für die Berechnung der erforderlichen Produktionsmittel bildeten die Arbeitsablaufpläne sowie die Betriebsmittelabmessungen. Im Rahmen dieses Beispiels erfolgt eine Beschränkung auf die Feststellung von Maschinen- und Flächenbedarf.

\section{Betriebsmittelbedarf}

Der qualitative Maschinenbedarf ergab sich nach der Aufstellung der Arbeitsablaufpläne. Zur Berechnung des quantitativen Betriebsmittelbedarfs wurde die in [2] erläuterte Berechnungsformel verwendet. Insgesamt wurden 30 Betriebsmittel untersucht, wobei sich für 13 Betriebsmittel Veränderungen gegenüber dem vorhandenen Potential ergaben. Durch Variation des Schichtfaktors und der damit verbundenen Erhöhung der vorhandenen Kapazität konnte der zunächst nach der Berechnungsvorschrift bestimmte Bedarf für 4 Betriebsmittel gesenkt werden, bei 5 Betriebsmitteln wude der bisherige Schichtfaktor beibehalten (siehe Abb. 3).

\begin{tabular}{|cccccc|}
\hline BM-Nr: Ist-Bestand & Ist-Schicht & $\begin{array}{r}\text { Neu-Bestand } \\
\text { (Berechnung) }\end{array}$ & Neu-Schicht & $\begin{array}{r}\text { Neu-Bestand } \\
\text { (Variation) }\end{array}$ \\
1 & 3 & 1 & 7 & 2 & 4 \\
2 & 2 & 1 & 8 & 2 & 4 \\
3 & $(1)$ & 1 & 4 & 1 & 4 \\
4 & $(1)$ & 1 & 3 & 1 & 3 \\
5 & $(5)$ & 1 & 33 & 2 & 17 \\
6 & 1 & 1 & 2 & 2 & 1 \\
7 & 4 & 2 & 3 & 2 & 3 \\
8 & 4 & 2 & 3 & 2 & 3 \\
9 & 2 & 2 & 3 & 2 & 3 \\
\hline
\end{tabular}

Abb. 3: Betriebsmittelbedarfsplan

Nach Bestimmung des Auslastungskoeffizienten für alle Betriebsmittel ergab sich für 4 Betriebsmittel eine sehr geringe Auslastung. Für diese Betriebsmittel wurden Überlegungen zur Auswärtsvergabe der Arbeitsgänge 
angestellt. Bei einer Anlage wäre eine solche Möglichkeit denkbar. Bei den uibrigen Fertigungseinrichtungen handelt es sich allerdings um Spezialmaschinen, die eigens für bestimmte Arbeitsvorgänge entwickelt wurden. Eine Fremdvergabe der Aufträge ist somit nicht möglich. In Abb. 4 sind die erforderliche Maschinenbelegungszeit TK und die verplanbare Maschinbelegungszeit TE fuir ausgewählte Betriebsmittel jeweils vor und nach Variation des Schichtfaktors gegenuibergestellt.
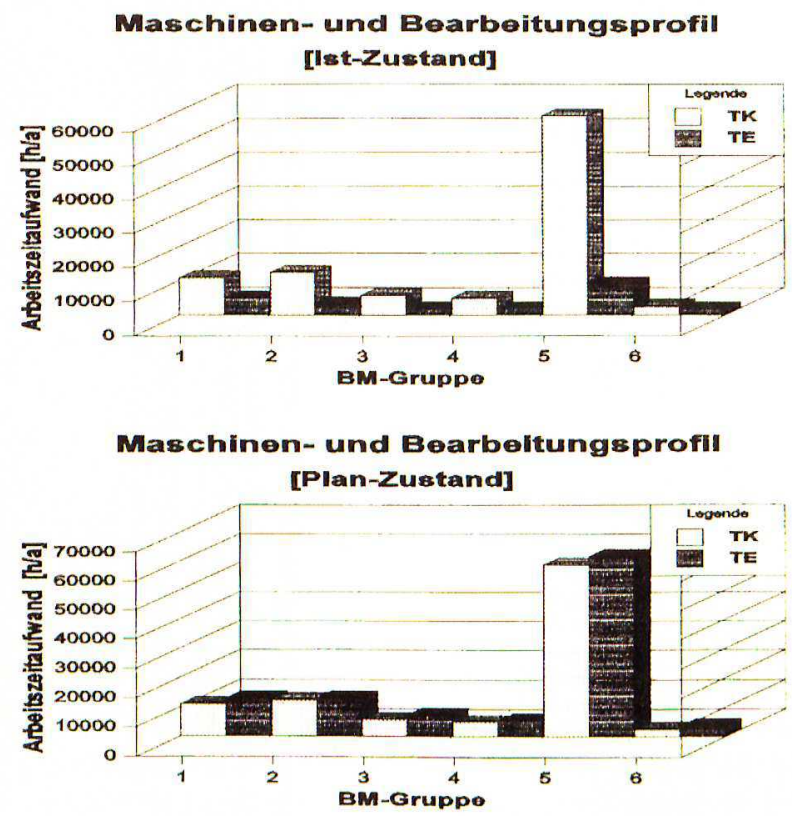

Abb. 4 Maschinen- und Bearbeitungsprofil (TK; TE)

\section{Flächenbedarf}

Grundlage für die Flächenermittlung bilden die Arbeitsplatzgrundflächen. Als Berechnungsvorschrift zur funktionalen Flächenermittlung wurde die in [2] beschriebene „KETTNER-Methode“ angewendet.

Insgesamt wurde für 56 Fertigungseinrichtungen bzw. Arbeitsplätze eine Flächenberechnung durchgeführt. Danach ergaben sich gegenüber dem Ist-Zustand folgende Veränderungen:

\begin{tabular}{|lrrr|}
\hline & IST-Zustand & Plan-Zustand & Veränderung \\
\hline Fläche insg. (Produktion) & $2108,51 \mathrm{~m}^{2}$ & $2016 \mathrm{~m}^{2}$ & $-4,4 \%$ \\
\hline Fertigung (inkl. Sanitär, & $1454,00 \mathrm{~m}^{2}$ & $1572 \mathrm{~m}^{2}$ & $+8,0^{\circ}$ \\
Pausenraum, Transportwege) & & & \\
Hauptlager & $442,51 \mathrm{~m}^{2}$ & $324 \mathrm{~m}^{2}$ & $-27,0^{\circ}$ \\
Medienversorgung & $212,00 \mathrm{~m}^{2}$ & $120 \mathrm{~m}^{2}$ & $-43,4^{\circ}$ \\
\hline
\end{tabular}

Abb. 5: Flächenbedarfsplan

Nach der Neuberechnung des Flächenbedarfes ergab sich eine Reduzierung der vorhandenen Fläche um ca. 4\%. Insbesondere für die Bereiche Hauptlager und Medienversorgung sind bei einer optimierten Flächennutzung Einsparungen im Flächenbedarf gegenüber dem Ist-Zustand erkennbar. Die Flächenberechnung bezieht sich nur auf die Produktionsfläche.

Der Flächenbedarf für den Entwicklungsbereich und die Verwaltung kann nach Kennzahlen bestimmt werden und kommt zu der Produktionsfläche hinzu.

\subsection{Materialflußgestaltung}

Innerhalb der Materialflußgestaltung wurden Festlegungen hinsichtlich Lagerwesen (Lagertyp und Lagerorganisation) sowie Förderwesen (Fördermittel und Förderhilfsmittel) getroffen. Ausschlaggebend waren dabei die Analyse des Lagergutes hinsichtlich Art und Menge soweit die in Punkt 4.1 getroffene Festlegung fuir die Produktionsstrategie. Die Ergebnisse dieser Untersuchung sind in Abb. 6 ersichtlich.

\begin{tabular}{|l|l|}
\hline Lagerwesen & $\begin{array}{l}\text { Lagerung von Einkaufsteilen/Halbfabrikaten/ } \\
\text { Kundendienstartikeln in Paletten-Flachregal- } \\
\text { lagern; Handbestiickung } \\
\text { Lagerung von Zwischenfabrikaten aus der } \\
\text { Produktion in Einschub-Regalanlage; Hand- } \\
\text { bestïckung } \\
\text { Lagerorganisation } \\
\text { Eöroilung der Lagerbereiche auf Basis einer } \\
\text { ABC-Analyse des Lagergutes (gezonte Lage- } \\
\text { rung) } \\
\text { Einfiihrung von BDE-Techniken } \\
\text { Kopplung von Lager und Produktion ïber In- } \\
\text { formationssystem zur Einbindung akuteller } \\
\text { Ist-Daten in Produktionsablauf }\end{array}$ \\
\hline Förderwesen & $\begin{array}{l}\text { Einsatz von Tischwagen innerhalb der Pro- } \\
\text { duktion und zwischen Produktion und Lager } \\
\text { fahrbare Transportwagen (höhenverstellbar) } \\
\text { zur Bedienung der Regalsysteme im Lager } \\
\text { Hubwagen zum Transportieren schwerer La- } \\
\text { sten im Lagerbereich } \\
\text { einheitliches Föderhilfsmittelsystem in Form } \\
\text { von Materialflußkästen mitverschiedenarti- } \\
\text { gen Einlagen zum Schutz des Fördergutes } \\
\text { Lagerung der Teile in Flachpaletten }\end{array}$ \\
\hline
\end{tabular}

Abb. 6: Förder- und Lagerwesen

\subsection{Layoutplanung}

Die Entwicklung des Werkstatt-Layouts vollzieht sich in mehreren Stufen, die nachfolgend erläutert werden.

\section{Fertigungsorganisation und Bereichsbildung}

Durch die Zusammenfassung von Arbeitsplätzen wurden Funktionseinheiten (Bereiche) gebildet. Wesentliche Kriterien waren dabei die speziellen Bedingungen für die einzelnen Arbeitsplätze hinsichtlich Klima-, Schmutzund Schwingungseinwirkungen. Es wurden die Arbeitsplätze zu Bereichen zusammengefaßt, die gleiche oder ähnliche raumqualitative Bedingungen erfordern.

Ein weiteres Kriterium war die Festlegung von Prinzip und Art der Fertigung.

Nach Analyse des Produktionsprogramms und der Arbeitsablaufpläne ergab sich die Werkstattfertigung als wirtschafltichstes Fertigungsprinzip und die Serienfertigung mit Losbildung fuir kundenanonyme Fertigungsaufträge sowie die Einzelfertigung für kundenspezifische Fertigungsaufträge als günstigste Fertigungsart.

Dementsprechend ergaben sich die in Abb. 7 zusammengestellten Funktionseinheiten. Aus Gründen der Übersichtlichkeit werden in den nachfolgenden Abbildungen 
die hier aufgeführten Kennbuchstaben für die einzelnen Bereiche verwendet.

\begin{tabular}{|clc|}
\hline Lfd.Nr: & Bereich & Kennung \\
\hline 1 & Wandlerkopfbearbeitung I & A \\
2 & Schweißen & B \\
3 & Bohren, Vereinzeln & C \\
4 & Messung zur Kompensation, Eröterung & D \\
5 & Chemie & \\
6 & Endprüfung & \\
7 & Wandlerkopfbearbeitung II & G \\
& Lötmontage, Endmontage, Dauertest & \\
8 & Sandstrahlen & H \\
9 & Pressen & I \\
10 & Zentrallager & L \\
11 & Applikationswerkstatt & K \\
\hline
\end{tabular}

Abb. 7: Funktionseinheiten der Geräteproduktion

\section{Ideales Funktionsschema}

Im idealen Funktionsschema wurde die ablauf- und funktionsgerechte Zuordnung der Funktionseinheiten dargestellt. Im Mittelpunkt stand dabei die Schaffung güinstiger Materialflußverhältnisse.

Bedingt durch die hohe Fertigungstiefe und Produktvielfalt ergibt sich eine intensive Materialflußverkettung. Eine optimale Materialflußgestaltung war somit nicht möglich. Gegenläufigkeiten sowie Kreuzungen im Materiafluß ließen sich nicht vermeiden.

Die in Abb. 8 dargestellte Variante stellt die unter den gegebenen Umständen günstigste Lösung dar.

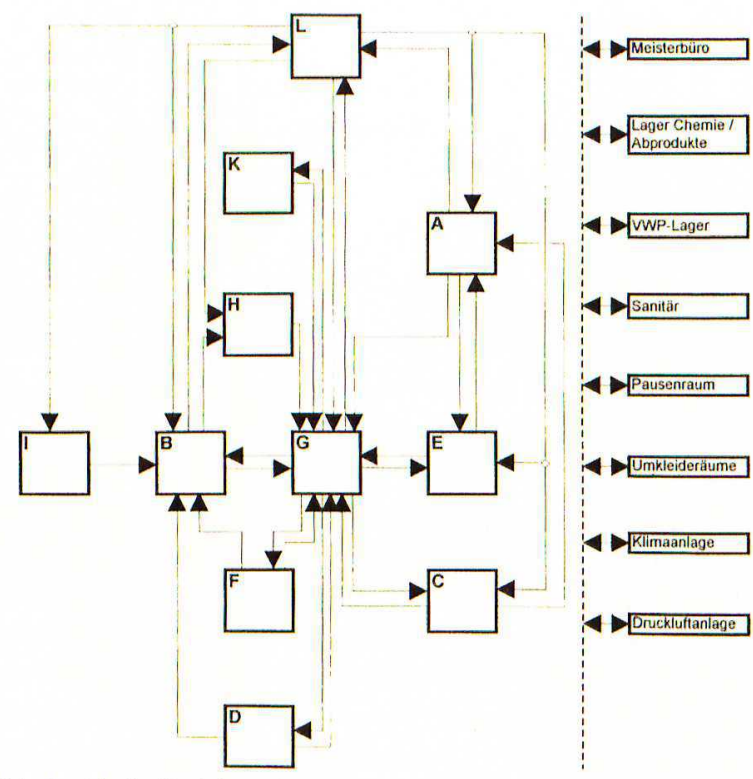

Abb. 8 Ideales Funktionsschema

Flächenmaßstäbliches Funktionsschema

Bezugnehmend auf die unter dem Punkt Bedarfsplanung ermittelten Flächenwerte fuir die einzelnen Arbeitsplätze wurden die gebildeten Funktionseinheiten maßstäblich aufgezeichnet.

Die Anordnung der einzelnen Funktionseinheiten entspricht dabei weitestgehend dem idealen Funktionsschema (Abb. 9).

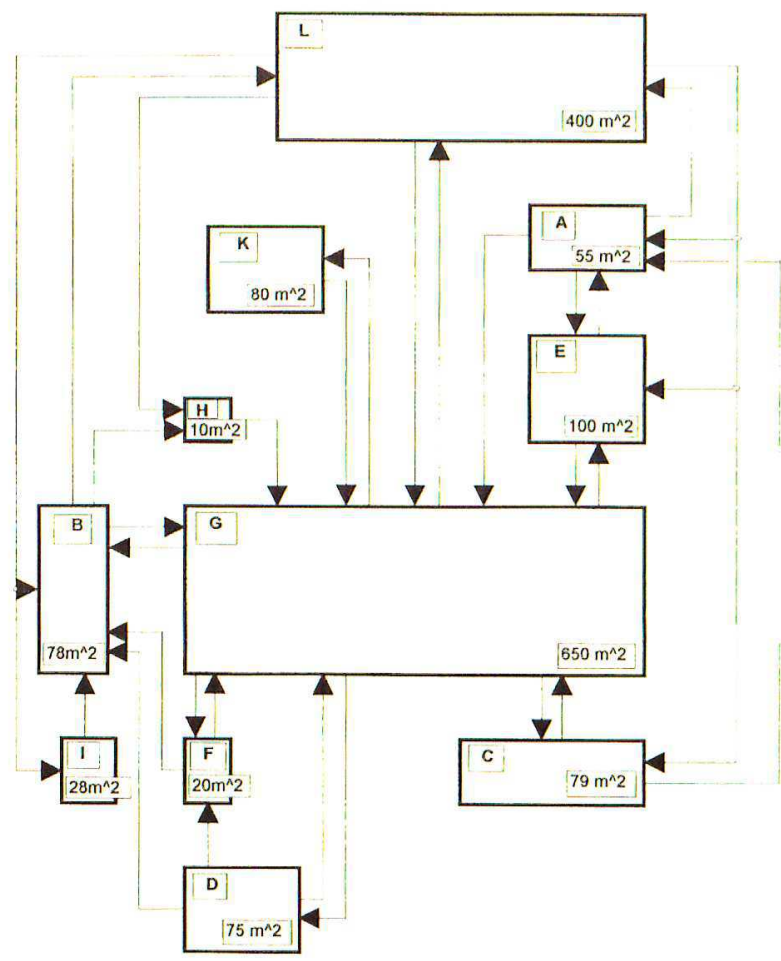

Abb. 9 Flächenmaßstäbliches Funktionsschema

\section{Beziehungsoptimierung}

Um die Anordnung der Funktionseinheiten hinsichtlich der bestehenden Materialflußintensität zu optimieren, wurde eine Transporttabelle in Form einer Von-NachMatrix erstellt (Abb. 10). Hieraus sind die zwischen den einzelnen Bereichen zu transportierenden Mengen in Stiick pro Jahr angegeben. Grundlage hierzu bildeten dabei die im Produktionsprogramm enthaltenen Erzeugnisstiickzahlen und die Arbeitsablaufschemata.

\begin{tabular}{|c|c|c|c|c|c|c|c|c|c|c|c|}
\hline $\begin{array}{l}\text { von/ } \\
\text { naach }\end{array}$ & A & B & C & D & E: & $\mathrm{F}$ & G & $\mathrm{H}$ & 1 & K & I. \\
\hline A & - & & & & 372 & & 9402 & & & & 1000 \\
\hline B & & - & & & & & 18900 & 2200 & & & 5750 \\
\hline C & 119 & & - & & & & 9219 & & & & \\
\hline D & & 2200 & & - & & 3550 & 3550 & & & & \\
\hline E: & 288 & & & & - & & 2836 & & & & \\
\hline F & & 2200 & & & & - & 4550 & & & & \\
\hline G & & 14850 & 527 & 6250 & 619 & 3200 & - & & & 2200 & 24950 \\
\hline H & & & & & & & 5750 & - & & & \\
\hline I & & 9300 & & & & & & & - & & \\
\hline K & & & & & & & 2200 & & & - & \\
\hline L. & 16917 & 25150 & 119 & & 2200 & & 34909 & 2550 & 9300 & & - \\
\hline
\end{tabular}

\section{Ideal-Layout}

Aufbauend auf dem idealen und dem flächenmaßstäblichen Funtkionsschema sowie der erstellten Beziehungsmatrix wurde das Ideal-Layout für den Fertigungskomplex entwickelt (Abb. 11). Die Pfeile kennzeichnen dabei die Materialflußbeziehungen zwischen den einzelnen Bereichen. Das Ideal-Layout erfüllt in erster Linie die Forderung nach einem möglichst optimalen Materialfluß unter Beachtung der Materialflußintensität.

\section{Real-Layout}

Der bisher erstellte Ideal-Zustand bezüglich der Anordnung der einzelnen Fertigungsbereiche zueinander muß im Rahmen der Realplanung an vorhandene bauliche Gegebenheiten und Restriktionen angepaßt werden. 


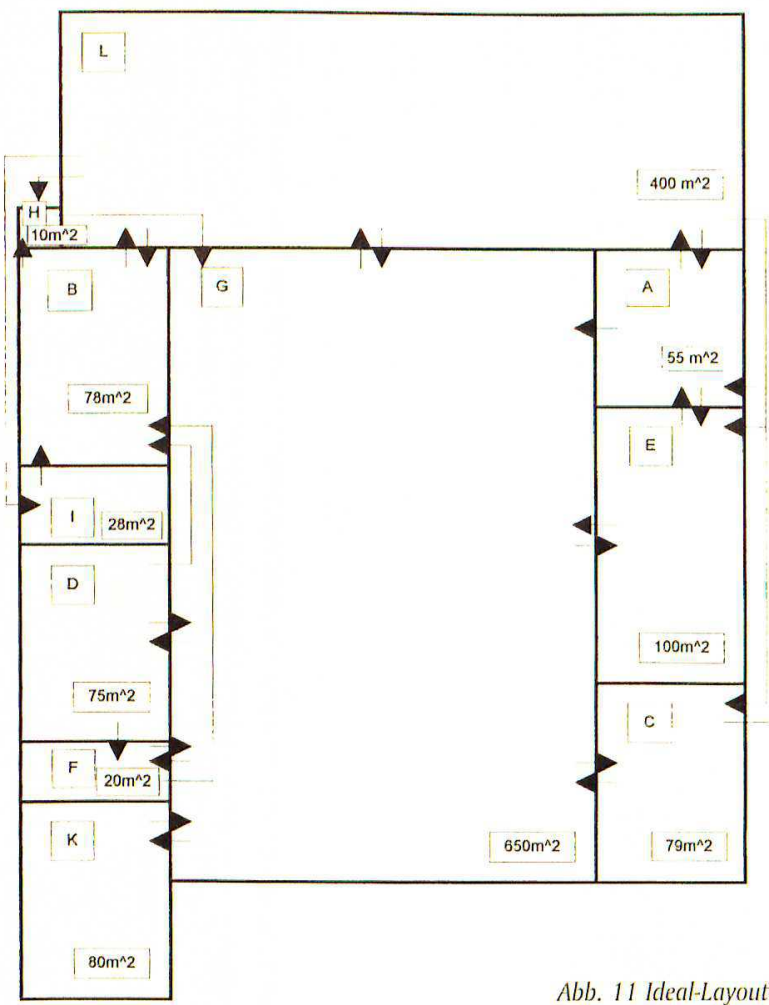

Da zum aktuellen Planungszeitraum keine konkreten Angaben zu bestehenden Restriktionen (Grundstiick, Gebäude) vorlagen, beschränkte sich die Realplanung auf die Auswahl eines geeigneten Gebäudetyps.

Bei dem ausgewählten Baukörper handelt es sich um einen Flachbau mit waagerechter Dachfläche, der folgende Merkmale aufweist:

- ebenerdig

- Grundfläche: $2016 \mathrm{~m}^{2}$

- Hallenspannweite: $36 \mathrm{~m}$

- Feldweitenhöhe: $4 \mathrm{~m}$

- Hallenhöhe: $\quad 4,20 \mathrm{~m}$

Die Maßangaben lehnen sich an die Vorzugsmaße für Flach- und Hallenbauten nach ISO-Norm an.

Im nächsten Schritt wurde nun versucht, den hinsichtlich Flächenanordnung und Materialfluß idealen Zustand an diesen ausgewählten Gebäudetyp anzupassen.

Restriktionen ergaben sich insbesondere durch den ausgewählten Gebäudetyp und die Bereichsverträglichkeit. Dazu entstanden meherere Layoutvarianten, die anschließend bewertet wurden.

Für das vorliegende Beispiel stehen drei unterschiedliche Grob-Layout-Varianten zur Auswahl (Abb. 12 bis Abb. 14). Ein bewertender Vergleich der Varianten wurde mit Hilfe der Nutzwertanalyse (Abb. 15) durchgeführt. Danach ist die Variante 3 die vorteilhafteste Grob-LayoutVariante.

\subsection{Bewertung der Lösungsvariante}

Abschließend soll die nach der Umgestaltung des Fertigungskompelxes entstandene Situation mit der Ausgangssituation verglichen werden.

In Auswertung der Abb. 16 ist eine deutliche Optimierung des Materialflusses zu erkennen. Dies wurde ins-

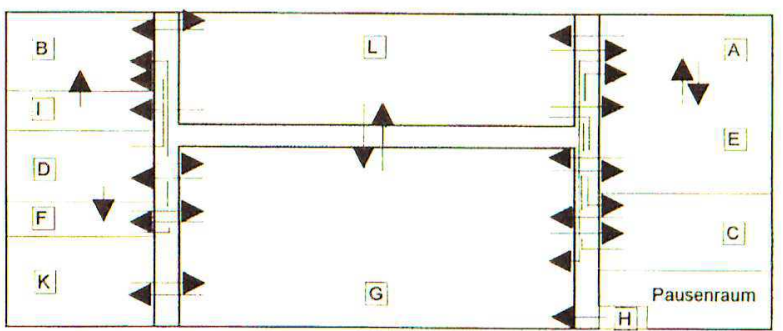

Abb. 12 Grob-Layout der Produktion, Variante 1

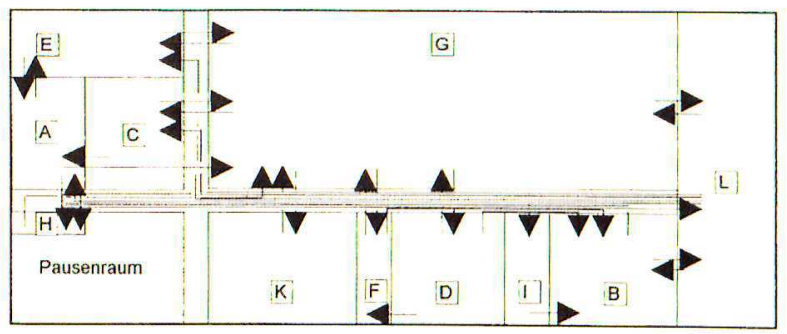

Abb. 13 Grob-Layout der Produktion, Variante 2

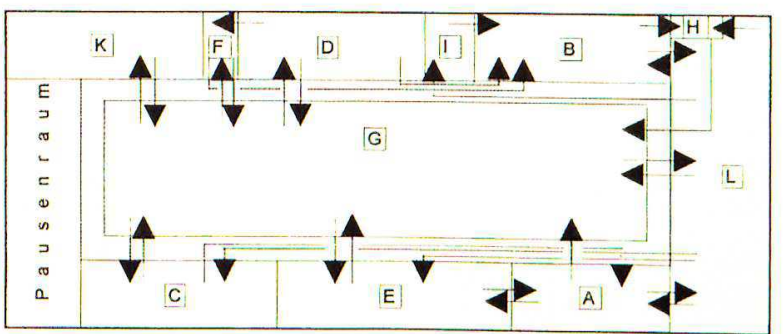

Abb. 14 Grob-Layout der Produktion, Variante 3

\begin{tabular}{lrrrr}
\hline Bewertungskriterium & Gewicht & Variante 1 & Variante 2 & Variante 3 \\
\hline Gebäudeinterner Materialfluß & 30 & 7210 & 260 & 8240 \\
Bereichsbildung & 20 & 8160 & 8160 & 8160 \\
Externer Materialfluß & 12 & 672 & 784 & 784 \\
Erweiterungsmöglichkeit & 12 & 448 & 448 & 448 \\
Produktionsmittel & & & & \\
(Aufstellung, etc.) & 8 & 432 & 540 & 648 \\
Flächennutzung & 10 & 550 & 440 & 660 \\
Gebäudeform & 8 & 648 & 648 & 648 \\
\hline Summe & 100 & 620 & 480 & 688 \\
Rangfolge & & 2 & 3 & 1
\end{tabular}

Abb. 15: Bewertung und Rangfolge der Grob-Layout-Varianten

besondere durch die Neuordnung der Funktionsbereiche erreicht, deren Anzahl sich von ehemals 15 auf 11 verringerte.

Die Fertigung und das Lager befinden sich in einer Ebene, wodurch sich der Transportaufwand erheblich reduzieren läßt.

Da sowohl der Wareneingang als auch der Warenausgang (Verpackung) räumlich zusammengefaßt wurden, besteht nur an einer Gebäudeseite eine Schnittstelle zum externen Materialfluß.

Unter Beachtung der erstellten Transportmatrix wurden die Bereiche mit einer hohen Materilaflußintensität so zueinander angeordnet, daß sich möglichst kurze und geradlinige Transportwege ergeben.

Der Betriebsmittel- und Flächenbedarf wurde an die für den kommenden Planzeitraum zu erwartenden Erzeugnistypen und -stiickzahlen angepaßt. Bei entsprechendem Flächenmehrbedarf ist eine Erweiterung jeweils an den Längsseiten des Gebäudes möglich. Innerhalb des 


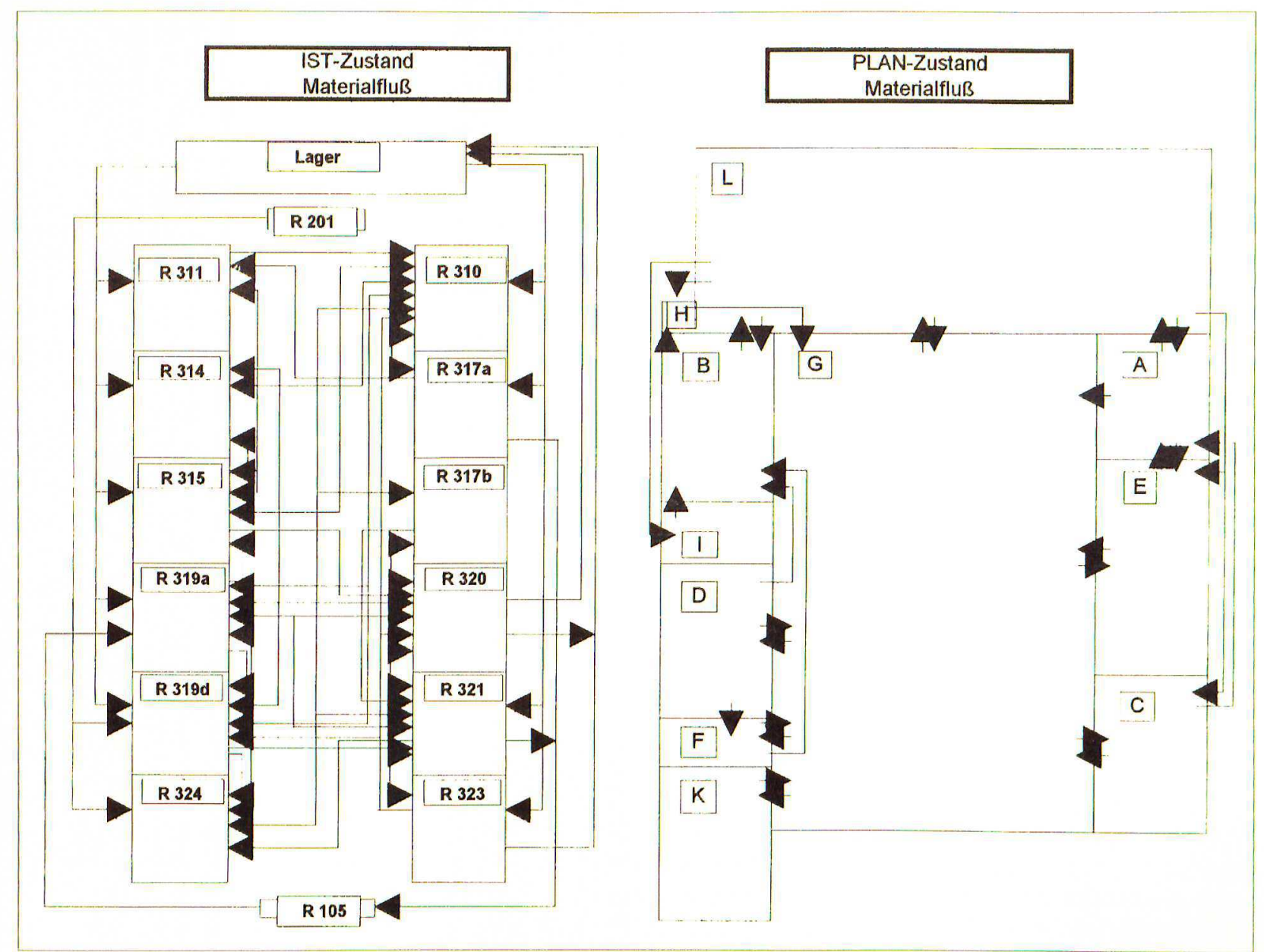

Abb. 16 Gegeniiberstellung Ist- und Plan-Zustand Materialfluß

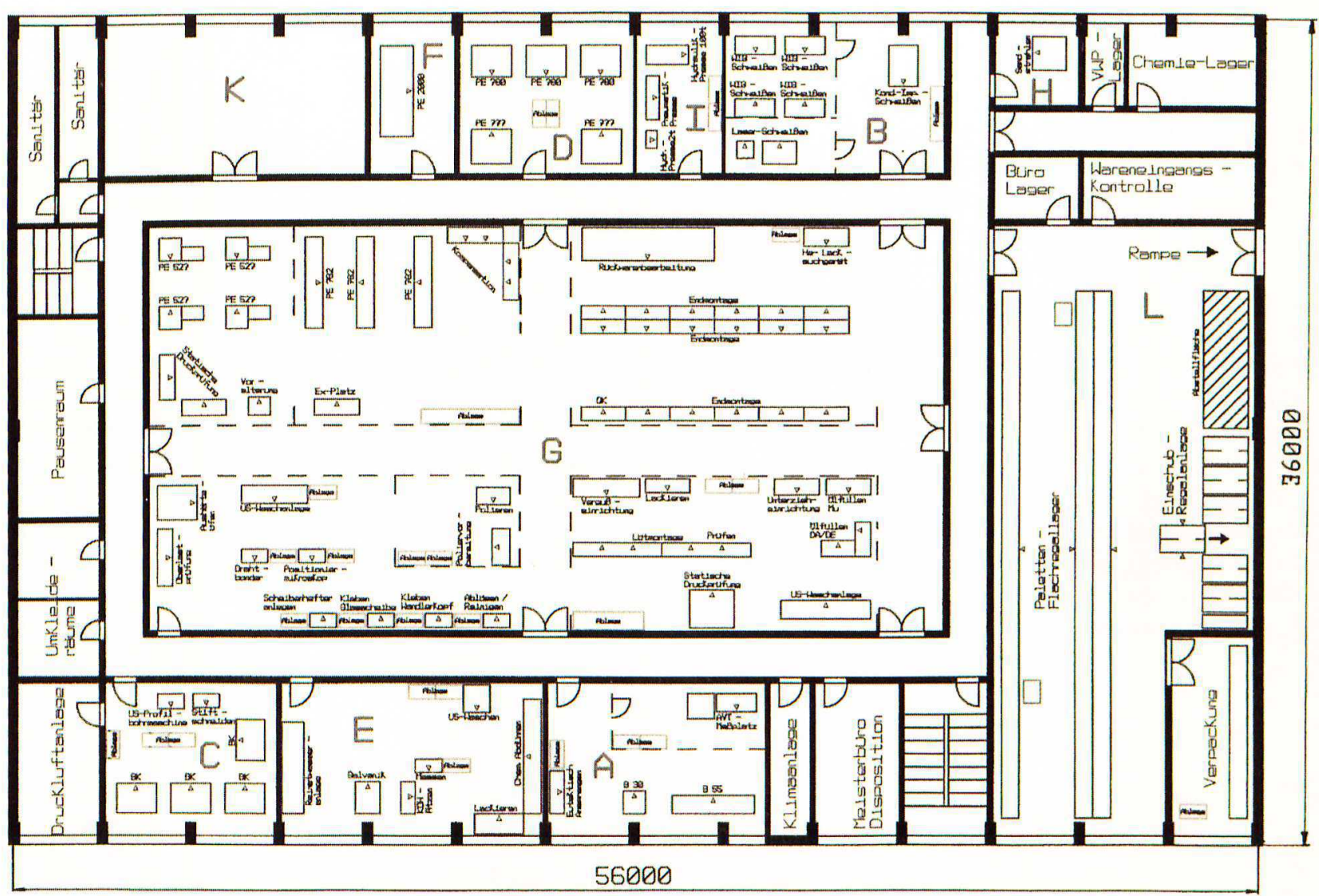

Abb. 17 Fein-Layout des Fertigungskomplexes 
Bereiches G stehen zusätzliche Nutzflächen zur Verfuigung.

Durch Variation des Schichtfaktors können die derzeit vorhandenen Betriebsmittel effektiver ausgenutzt werden.

Die Auswahl des Lagertyps und der Lagerorganisation entspricht der zur Zeit zu lagernden Menge an Artikeln und deren Beschaffenheit.

Von einer Automatisierung des Lagerwesens wurde Abstand genommen, da auf Grund der vorhandenen Materialflußintensität keine effektive Nutzung einer solchen Anlage möglich wäre.

Für die Bestimmung von Fördermitteln und Förderhilfsmitteln waren in erster Linie die Art und die Eigenschaften der Teile entscheidend. So besteht die Hauptfunktion der Förderhilfsmittel vor allem im Schutz der Teile gegen physikalische und chemische Einwirkungen.

Mit der Erstellung des Fein-Layouts (Abb. 17) kann die Planungsaufgabe als erfüllt angesehen werden.

Unter Voraussetzung einer ständigen Überpriifung und Konkretisierung der Planungsdaten im Planungsablauf bildet das Fein-Layout die Grundlage für die Realisierung des Planungsvorhabens.

\section{Abschlußbetrachtung}

Die Lösung der vorliegenden Fabrikplanungsaufgabe war unter Verwendung des in Abb. 1 dargestellten systematischen Planungsablaufes möglich. Eine Anwendung dieser Systematik fuihrte zu Handlungstätigkeiten und Konsequenzen hinsichtlich der Geräteproduktion. Die enthaltenen Ergebnisse sind aber als Ideal-Lösungen zu betrachten und muissen an die jeweils gegebenen Bedingungen angepaßt werden. Erst dann erhält man reale Resultate, die in der Praxis umsetzbar sind. Trotzdem ist die Durchfuihrung einer kompromißlosen Idealplanung in jedem Fall erforderlich, da sie einen objektiven Maßstab fuir die Realplanung bildet.

Unzulänglichkeiten der realen Lösung sind zu erkennen und gefährlichen Selbsttäuschungen kann somit vorgebeugt werden.

An dieser Stelle sei nochmals darauf hingewiesen, daß der Phase der Ist-Zustands-Aufnahme und -Bewertung eine außerordentliche Bedeutung zukommt. Werden in dieser Planungsphase wichtige Aspekte iibersehen oder nicht beachtet, kann sich die erstellte Lösungsvariante als nicht umsetzbar herausstellen und muß neu erarbeitet werden.

\section{Literaturverzeichnis}

[1] A. Guhl: Diplomarbeit, Thema: Analyse und Neugestaltung des Fertigungskomplexes „Druckmeßtechnik“ (1994); Betreuer: Prof. Dr. Grundig, TFH Wildau, FB Maschinenbau

[2] Kettner/Schmidt/Greim: Leitfaden der systematischen Fabrikplanung, Carl Hanser Verlag München/ Wien 1984

[3] R. Jünemann: Materialfluß und Logistik, Springer Verlag Berlin/ Heidelberg 1989

[4] H.-P. Wiendahl: Betriebsorganisation, Carl Hanser Verlag München/Wien 1989

[5] Dolezalek, Warnecke: Planung von Fabrikanlagen, Springer Verlag Berlin/Heidelberg 1981

[6] VDI-Richtlinie 2689: Leitfaden für Materialflußuntersuchungen

\section{Verfasser}

Dipl.-Ing. (FH) Astrid Guhl

Technische Fachhoschule Wildau

Fachbereich Betriebswirtschaft/Wirtschaftsinformatik

Laboringenieurin

Tel. (0 33 75) 507-136 